\title{
Intratympanic gentamicin injection in Ménière's disease: our experience and outcomes
}

\author{
Barbara Filosa $^{1}$, Antonio Trusio ${ }^{2}$, and Giuseppe Malafronte ${ }^{1}$ \\ ${ }^{1}$ National Speciality Hospital San Giuseppe Moscati \\ ${ }^{2}$ Azienda Ospedaliera di Rilievo Nazionale e di Alta Specialita San Giuseppe Moscati
}

December 2, 2020

\begin{abstract}
Title: Intratympanic gentamicin injection in Ménière's disease: our twelve years' experience and outcomes. Objective: The aim of our study is to evaluate the effectiveness of intratympanic gentamicin injection(ITG) on vertigo control with reduced doses and its hearing effects. Study design: Retrospective study Materials and Method: The study was conducted at our Otolaryngology Department of AORN "S.G. Moscati" between January 2005 and January 2015 on 72 patients with disabling unilateral Meniere's disease treated with ITG. We use $0.2-0.3 \mathrm{~mL}$ of gentamicin sulfate at a concentration of $40 \mathrm{mg} / \mathrm{ml}$, injected into the affected ear through the posterior-inferior quadrant of the tympanic membrane. The procedure was carried out for three following days. Main outcome measures: vertigo control and hearing threshold changes after ITG treatment. Results: In the $98.6 \%$ of the patients $(\mathrm{n}=71)$ the ITG produced the full remission of the vertiginous symptoms. In the $91.6 \%$ of cases $(\mathrm{n}=66)$ a single treatment (three consequent injections) was sufficient to control vertigo, in the $5.5 \%$ of cases $(n=4)$ two treatments were necessary to control vertigo and in the $1.3 \%$ of patients $(\mathrm{n}=1)$ three treatments were necessary to control vertigo. In no case we have had hearing loss after ITG procedure. The pre-treatment pure tone average was $48 \mathrm{db}$. The post-treatment pure tone average was $49.2 \mathrm{db}$. This difference was no statistical difference. Conclusion: In this study we reported high vertigo control, long follow-up and no case of significant hearing worsening. We consider the three injections in the following three days with low doses of gentamicin a safe and valid treatment for Meniere's disease. Keyword : Meniere's disease, intratympanic gentamicin injection, vertigo control, hearing worsening, aminoglycoside ototoxicity.
\end{abstract}

\section{Introduction}

Meniere's disease(MD) is an idiopathic inner ear disorder characterized by episodic attacks of vertigo, fluctuating hearing loss, tinnitus, and aural fullness. MD exhibits a relapsing-remitting pattern, with episodic attacks terminated by periods of restitution to normal auditory and vestibular function. Additionally, auditory and vestibular function decline over time.Although the pathophysiologic mechanism of MD is well accepted, the underlying causes remains uncertain ${ }^{1}$. MD treatment is still objective of controversies in literature and it must be first based on a trustable diagnosis, as recommended by guidelines and classification of the AAO-HSN published in $1995^{2}$ and reviewed by the Equilibrium Committee in $2015^{3}$. MD has a considerable negative effect on patient's quality of life so current therapies are directed to prevent or reduce the frequency and severity of vertigo crises $^{4}$. Intratympanic injection of gentamicin(ITG) is considered the treatment of choice in patients with refractory disease ${ }^{1,4}$. However due to its possible hearing side effects there is still no general consensus on dose and timing of $\mathrm{ITG}^{4}$.

The aim of our study is to evaluate the effectiveness of intratympanic injection of gentamicin on vertigo control with close doses and its hearing effects.

Material and Methods 
The study was conducted at Otolaryngology Department of AORN"S.G.Moscati" between January 2005 and May 2015.We selected 72 consecutive patients(28 males and 44 females, mean age 49.5 years, range:21-78 years) with disabling unilateral Ménière's disease treated with ITG. Patients included in the study were not responsive to conservative therapies(lifestyle change, diuretics, salt-restricted diet, steroid or betahistine). Inclusion criteria for this study were: definite diagnosis of MD based on criteria of the 1995 Equilibrium Committee $^{2}$, lack of response to medical therapy for six months with invalidating vertigo attacks, unilateral $\mathrm{MD}$, satisfactory hearing in the contralateral ear, no previous treatment with surgical conservative procedures or intratympanic steroid injections, absence of hypersensitivity to aminoglycosides. All patients included in the study met the inclusion criteria. After a detailed explanation of the procedure, a written consent was obtained. To exclude other inner ear diseases, all patients were submitted to magnetic resonance with contrast. Before intratympanic treatment all patients underwent a neuro-otological examination, including evaluation of spontaneous, positional and positioning nystagmus, head shaking test, subjective visual vertical test, Romberg and stepping test. A caloric test was used to evaluate the vestibular semicircular canal excitability. Otomicroscopic examination was performed to evaluate tympanic membrane. All patients underwent to tympanometry and pure tone audiometry. Pure tone average(PTA) was calculated on air conduction thresholds at 500, 1000, 2000 and 3000dB. Before treatment all patients filled out a questionnaire about number of vertigo, so to calculate a monthly rate of vertigo. We use gentamicin sulfate at a concentration of $40 \mathrm{mg} / \mathrm{ml}$, the intratympanic injection was performed with a $25 \mathrm{G}$ spinal needle in our ambulatory. Without local anesthesia, $0,2-0,3 \mathrm{~mL}$ of gentamicin was injected into the affected ear through posterior-inferior quadrant of tympanic membrane. Patient was instructed to maintain a supine position with the head 30 degree tilted toward the healthy side avoiding to swallow for at least $30 \mathrm{~min}$. The same procedure was carried out the following two days. After treatment audiometric revaluation was performed at 3 and 7 days after the third injection. In absence of vertigo patients were revaluated after 1-3-6-12 months and then every year. The follow up ranged from 2 to12 years(mean follow-up 7 years). The aim of the study was to evaluate hearing threshold changes and vertigo control after ITG treatment. The t student test was used to compare outcomes. For quantitative variables, descriptive statistics were computed as mean and Standard Deviation(SD) or median and Interquartile Range(IQR $25^{\text {th }}$ to $75^{\text {th }}$ percentiles), if they were not normally distributed, and as absolute frequency and percentage for qualitative variables. The reporting guideline followed in this study was STROBE statement.

\section{Results}

During the procedure patients complained of a bearable pain, comparable to an intramuscular injection. In the 6 months prior to treatment, patients reported a mean frequency of six vertigo attacks per month(range 2-12). Pre-treatment median PTA was $48 \mathrm{~dB}(\mathrm{IQR} 44-52)$ and median unilateral caloric weakness was $42 \%$ (IQR 32-57). In $98.6 \%$ of the patients $(\mathrm{n}=71)$ the ITG produced full remission of vertiginous symptoms. In $91.6 \%$ of $\operatorname{cases}(\mathrm{n}=66)$ a single treatment(three consequent injections) was sufficient to control vertigo, in $5.5 \%$ of $\operatorname{cases}(\mathrm{n}=4)$ two treatments were necessary to control vertigo( in 2 patients 1 year after first treatment; in 1 patients 2 year after first treatment; in 1 patient 9 year after first treatment) and in $1.3 \%$ of patients $(\mathrm{n}=1)$ three treatments were necessary to control vertigo( 4 years and 6 years after first ITG). Number of gentamicin injections applied to achieve vertigo control is shown in Fig.1. In the unsuccessful patient we have repeated ITG treatment five times (1, 3, 6 and 7 years after first ITG) without full control on the vertigo symptoms and, however, without significantly hearing impairment( pre-treatment $\mathrm{PTA}=51.25$; post-treatment $\mathrm{PTA}=52.5$; t-value $=0.65$; The $p$-value is 0.26 ). In no case we have had hearing loss after ITG procedure. Post-treatment PTA was 49.2dB(IQR 44-54). The difference between pre and post treatment PTA was no statistical difference at $\mathrm{p}<0.05$ ( $\mathrm{t}$-value $=-12.1 ; \mathrm{p}$-value $<.00001)$. Mean post-treatment unilateral caloric weakness was $78 \%$ (IQR 48-88). The difference between pre- and post-treatment value was statistically significant at $\mathrm{p}<0.05(\mathrm{t}$-value $=0.40 ; \mathrm{p}$-value $=0.34)$. In all cases the same procedure was performed (three following daily ITG of $40 \mathrm{mg} / \mathrm{ml}$ concentration). All patients of the study group complained imbalance after gentamicin procedure which resolved 1 to 2 months after the IGT without a specific vestibular rehabilitation, rather we recommend patients to practice light physical activity (such as walking) as soon as possible.

\section{Discussion}


Nowadays there is an international consensus on diagnosis of MD while there is still no general consensus on the most appropriate treatment to be adopted ${ }^{1,4}$. Although treatment of medical refractory unilateral MD with IT aminoglycoside injections has nowadays a standard therapy, there is still no consensus on the doses and timing of administration ${ }^{4,5}$. The IT aminoglycoside injections was first introduced by Schuknecht using streptomycin and then by Lange using gentamicin. Gentamicin has now become the most widely used aminoglycoside for treatment of vestibular symptoms associated with MD. It's a relative selective vestibulotoxic aminoglycoside antibiotic that is preferentially taken up by type I hair cells of the vestibular neuroepithelium ${ }^{6}$. The ITG treatment was initially conceived as an ablative method with complete vestibular ablation to control vertigo ${ }^{1}$. However, with this approach there were serious cochleotoxic effects with great hearing loss risk ${ }^{1}$.Parnes reported $41,7 \%$ hearing worsening using high doses of gentamycin(3 daily injections for 4 days $)^{7}$. Similar results were reported by Cortsen who performed 3 times daily ITG for 3 days ${ }^{8}$. It is likely that the initial reversible effect of gentamicin on both the vestibule and cochlea turns eventually to an irreversible stage due to the accumulation of consecutive doses in the inner ear because of slow clearance of gentamicin ${ }^{9}$.It has been demonstrated that gentamicin is eliminated slowly from the inner ear and ototoxic effects of ITG were delayed 2-3 days after the last installation ${ }^{10,11}$.Low-dose treatment may produce sufficient loss of vestibular function to provide relief from vertigo, and with less risk of affecting the hearing level. Even though over the last decade there has been a general trend to conserve vestibular and consequently cochlear function in order to obtain vertigo control with a very low hearing risks, some physicians are still concerned about hearing loss.

In our study we used a low dose of gentamycin injected once daily for 3 consecutive days with high vertigo control $(98.6 \%)$ and no case of statistically significant hearing impairment. Similar results were reported by Quaglieri et al, who achieved $96.5 \%$ of total effective vertigo control with no cases of statistical hearing impairment ${ }^{11}$. We agree with Yetiser who states that titration methods or multiple injections on a daily basis can be used only in patients with profound or non-serviceable hearing, since these methods have significant incidence of hearing $\operatorname{loss}^{9}$. In our series with a long follow-up, we noticed that the used protocol (three injections in the following three days) provides effective vertigo control without risk of anacusia and almost no risk of hearing impairment. In one case of our series we have repeated the ITG treatment five times $(1,3,6$ and 7 years after the first ITG) without achievement the full control on the vertigo symptoms but,however, without significantly hearing impairment. It is likely that if the patient had no hearing damage on the first treatment he probably does not have a genetic predisposition for aminoglycoside ototoxicity ${ }^{12}$. We are aware that the limit of our work is that it is a retrospective study. For this reason, at the end of 2015 we have started a new prospected study to check if a further reduction of the ITG administration times gives the same satisfactory results in vertigo control.

\section{Conclusion}

Taking into account that the great limit of this study is that it is an observational retrospective study, we reported high vertigo control, long follow-up and no case of significant hearing improvement. For these reasons we consider the three injections in the following three days with low doses of gentamicin as a safe and valid treatment for Meniere's disease.

\section{References}

1. Yaz F, Ziylan F, Smeeing DPJ, Thomeer HGXM. Intratympanic Treatment in Menière's Disease, Efficacy of Aminoglycosides Versus Corticosteroids in Comparison Studies: A Systematic Review. Otol Neurotol. 2020 Jan;41(1):1-10.

2. Committee on hearing equilibrium guidelines for the diagnosis evaluation on therapy in Ménière's disease. Otolaryngol Head and Neck Surg 1995; 113:181-5.

3. Lopez-Escamez JA, Carey J, Chung WH, et al. Diagnostic criteria for Ménière's disease. J vest Res 2015;25:1-7.

4. Basura Gj, Adams ME, Monfared A, et al. Clinical Practice Guideline: Meniere's Disease. Otolaryngol Head Neck Surg. 2020:162(2_suppl):S1-S55 doi: 10.1177/0194599820909438.

5. Nevoux J, Barbara M, Dornhoffer J, et alt. International consensus (ICON) on treatment of Ménière's 
disease. Eur Ann Otorhinolaryngol Head Neck Dis. 2018 Feb;135(1S):S29-S32.

6. Lyford-Pyke S, Vogelheim C, Chu E, et al. Gentamicin is primarily localized in vestibular type I hair cells after intratympanic administration. J Assoc Res Otolaryngol 2007;8(4): 497-508.

7. Parnes LS, Riddell D. Irritative spontaneous nystagmus following intratympanic gentamicin for Menière's disease. Laryngoscope. 1993 Jul;103(7):745-9.

8. Corsten M1, Marsan J, Schramm D et alt. Treatment of intractable Menière's disease with intratympanic gentamicin: review of the University of Ottawa experience. J Otolaryngol. 1997 Dec;26(6):361-4.

9. Yetişer S. Intratympanic Gentamicin for Intractable Ménière's Disease - A Review and Analysis of Audiovestibular Impact. Int Arch Otorhinolaryngol. 2018 Apr;22(2):190-194.

10. Magnusson M, Padoan S, Karlberg M, et alt. Delayed onset of ototoxic effects of gentamicin in patients with Meniére's disease. Acta Otolaryngol Suppl. 1991;485:120-2.

11. Quaglieri S, Gatti O, Rebecchi E, et alt. Intratympanic gentamicin treatment 'as needed' for Meniere's disease. Long-term analysis using the Kaplan-Meier method.Eur Arch Otorhinolaryngol. 2014 Jun;271(6):1443-9. doi: 10.1007/s00405-013-2597-7

12. Higashi K. Unique inheritance of streptomycin-induced deafness. Clin Genet 1989;35:433-436.

Data Availability StatementData are available on request from the authors. The data that support the findings of this study are available from the corresponding author upon request, with the agreement of our health management department.

\section{Figure Legend}

Fig. 1. Number of treatments to achieve vertigo control. Each treatment consists of one injection per day for 3 consecutive days.

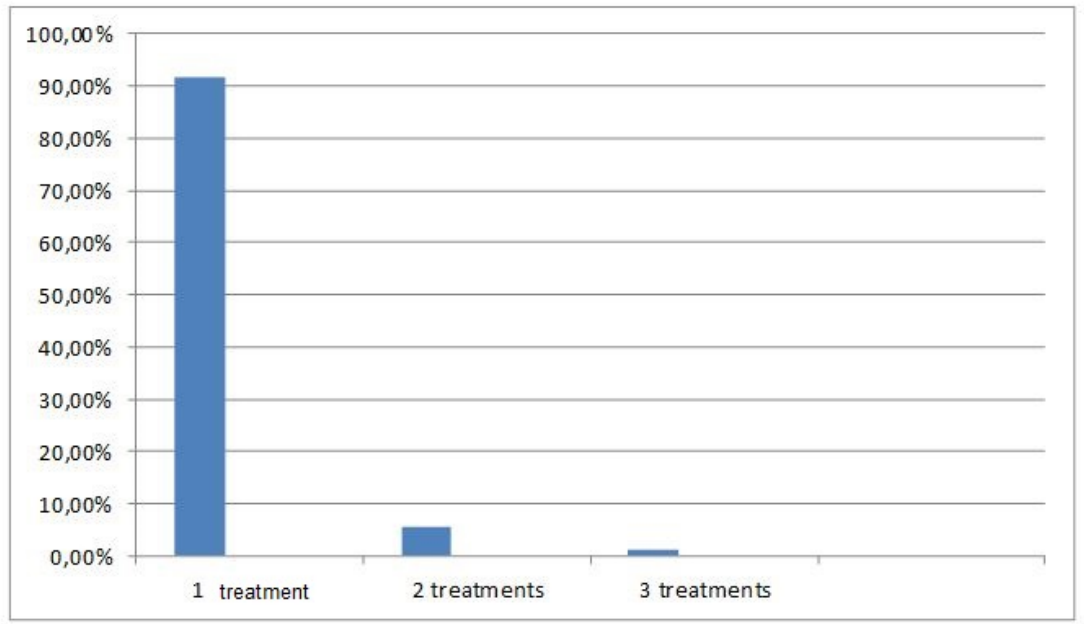

